University of Nebraska - Lincoln

DigitalCommons@University of Nebraska - Lincoln

3-15-1985

\title{
Derivation of discrete invariances $(T, C$, and $P)$ and the connection between spin and statistics in topological particle theory
}

C. E. Jones

University of Nebraska-Lincoln

Paul Finkler

University of Nebraska-Lincoln, pfinkler1@unl.edu

Follow this and additional works at: https://digitalcommons.unl.edu/physicsfinkler

Part of the Physics Commons

Jones, C. E. and Finkler, Paul, "Derivation of discrete invariances ( $T, C$, and $P$ ) and the connection between spin and statistics in topological particle theory" (1985). Paul Finkler Papers. 13.

https://digitalcommons.unl.edu/physicsfinkler/13

This Article is brought to you for free and open access by the Research Papers in Physics and Astronomy at DigitalCommons@University of Nebraska - Lincoln. It has been accepted for inclusion in Paul Finkler Papers by an authorized administrator of DigitalCommons@University of Nebraska - Lincoln. 


\title{
Derivation of discrete invariances $(T, C$, and $P$ ) and the connection between spin and statistics in topological particle theory
}

\author{
C. E. Jones and P. Finkler \\ Behlen Laboratory of Physics, University of Nebraska-Lincoln, Lincoln, Nebraska 68588
}

(Received 17 August 1984)

\begin{abstract}
For purely hadronic processes, the standard connection between spin and statistics as well as separate invariances under charge conjugation, parity, and time reversal are shown to be consequences of self-consistency in topological particle theory.
\end{abstract}

\section{INTRODUCTION}

In the preceding work ${ }^{1}$ (referred to hereafter as FJ) the phases for zero-entropy terms in the topological expansion were determined through systematic enforcement of the requirements of pole factorization, Hermitian analyticity and crossing symmetry. In this paper we investigate the spin and statistics properties of these zero-entropy terms and also their invariance properties under parity, timereversal, and charge-conjugation transformations. All these properties are shown to follow from self-consistency considerations and a knowledge of the amplitude phases derived in FJ.

In Sec. II the theory is shown to obey the standard spin and statistics relations. The proof differs considerably from an $S$-matrix proof of spin and statistics given a number of years ago by Stapp, ${ }^{2}$ since these topological amplitudes are not unitary at the zero-entropy level. We also show (Secs. III, IV, and V) that the zero-entropy sum is separately invariant under parity, time-reversal, and charge-conjugation transformations. The zero-entropy level and higher corrections arising from connected sums of these zero-entropy terms represent hadronic processes so we have been able to prove the existence of the standard discrete symmetries for strong interactions as well as the standard statistics. It should be emphasized that these symmetries were not built into the theory. Only requirements of self-consistency, pole factorization, crossing symmetry and Hermitian analyticity are used together with a standard discontinuity equation which embodies the dynamics. Again these results should be contrasted with Stapp's original work $^{2}$ where the product of these three symmetries was shown to hold for the full unitary $S$ matrix.

The discussion of the discrete invariances is carried out in the framework of the topological bootstrap theory. After defining the invariances we simply investigate whether the amplitude for a process has the same absolute value as the appropriately transformed process. It is not therefore necessary to define the action of these symmetry transformations on individual incoming or outgoing state vectors. Thus many tiresome phase questions are avoided and yet any physically observable phases (such as those associated with intrinsic parities ${ }^{3}$ ) are unambiguously evident.
The study of electroweak processes within the framework of the topological theory ${ }^{4-6}$ shows that the amplitudes for such processes appear above the zero-entropy level where the discrete symmetries no longer hold separately. The topological theory thus provides a basis for understanding the presence of the separate discrete symmetries in certain (strong) processes as well as their absence in other (weak) processes.

\section{SPIN AND STATISTICS FOR ZERO-ENTROPY AMPLITUDE}

Each term in the zero-entropy sector of the topological expansion corresponds to a different self-consistent zeroentropy amplitude for the particular scattering process being considered. A process is defined by giving particle types, momenta, and spin states for the process. The individual terms which are summed in the expansion correspond to topologically inequivalent zero-entropy amplitudes for the process. This summation involves two parts: (1) a sum over all possible spin-patch orientations for a given graph, (2) a sum over inequivalent graphs for the same process. For a particular process, we shall represent these two sums over zero-entropy amplitudes in the topological expansion in the following way:

$\sum_{\left\{z_{A} \cdots z_{K}\right\}} \sum_{i} A^{z_{A} \cdots z_{K}}\left(P_{i}(A \cdots K)\right) \equiv A(A \cdots K)$,

where as in FJ the patch structure for the amplitude is denoted $\left\{z_{A} \cdots z_{K}\right\}$ and the variables $A, \ldots, K$ denote the momentum, spin state, and flavor content (or type) of each particle in the process. The summation over $i$ in (2.1) is over inequivalent graphs and $P_{i}$ is an appropriate permutation of the variables in each case. As indicated in Sec. II of FJ (see also Stapp ${ }^{7}$ and Chew and Poénaru ${ }^{8}$ ) the graphs to be included in the sum over $i$ in (2.1) are those which differ from each other by more than just a cyclic permutation of the variables. The cyclic permutations of variables associated with a given graph give rise to equivalent amplitudes differing at most by a phase. This is the phase denoted $\tau$ in FJ which depends only on the cyclic order of the variables. A central issue of this paper is the determination of which permutation (more specifically which cyclic permutation) is to be used for each

(c) 1985 The American Physical Society 
term in the $i$ sum of (2.1). This will in turn determine the spin-statistics properties of the amplitudes. Of course, one of the amplitudes in (2.1) can be specified with an arbitrary cyclic order for its variables, but after that the cyclic order of variables for the remaining amplitudes is not arbitrary.

Let us first focus on the permutation summation over $i$ in (2.1). Since the graphs as discussed in FJ are made up of quark and diquark lines we can conclude that amplitudes for processes that do not involve any baryons or antibaryons must involve either all mesons or all baryonia. In these two special cases the $\tau$ factor is the same for each cyclic order of the variables for each graph so the $i$ sum of (2.1) has no ambiguity. Up to a cyclic permutation the graph determines the permutation $P_{i}$ and the amplitude is invariant under cyclic permutations.

We may readily verify that for amplitudes of the type just mentioned-all mesons or all baryonia-the mesons and the baryonia obey Bose statistics. To see this, let us suppose that particles $B$ and $J$ are the same type. Then the sum of amplitudes in (2.1) must consist of pairs of terms with $B$ and $J$ interchanged. The sum (2.1) is thus clearly unchanged when the variables $B$ and $J$ are interchanged implying Bose statistics.

When baryons and antibaryons are present in the amplitude the overall sign or $\tau$ factor for the amplitude will in general depend upon which cyclic permutation of variables is used for a given graph. We recall from FJ that $\tau=+1$ if the amplitude begins its cyclic order with a diquark line and $\tau=-1$ if it begins its cyclic order with a single quark line. It is useful for this discussion to introduce an amplitude with abbreviated variables of the type shown in Fig. 1. The external lines in Fig. 1 actually denote clusters of particles consisting of a single baryon or antibaryon with an arbitrary number of mesons or baryonia. The dot in Fig. 1 designates the beginning of the cyclic order for the amplitude, so in this example we would write the amplitude $A(1,2,3, \ldots, \alpha, \beta, \gamma, \ldots)$. The variables $1,2,3, \ldots, \alpha, \beta, \gamma, \ldots$ designate the momenta, spin states, etc., for the baryons and antibaryons within the cluster. The variables describing the mesons and baryonia which may be present in the various clusters have been suppressed. This streamlined notation is especially serviceable for our purposes because we are interested mainly in the $\tau$ phase of a given amplitude as a function of the cyclic order of the variables as given by the dot in Fig. 1. The $\tau$ phase for the amplitude is completely specified by indicating between which two external lines

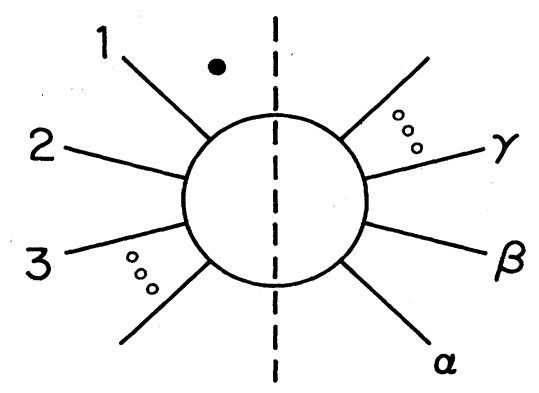

FIG. 1. Amplitude with baryon or antibaryon clusters.

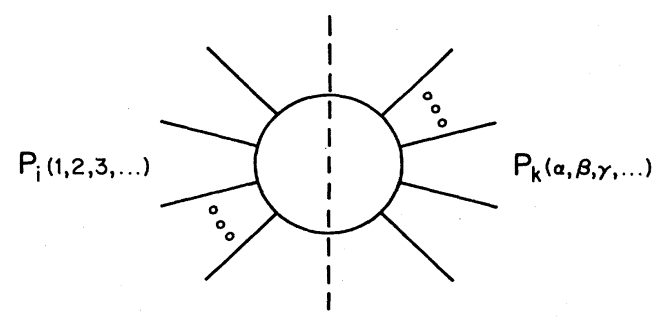

FIG. 2. Same process as Fig. 1 but with particle lines permuted.

the dot is placed in Fig. 1. From the way $\tau$ is determined ( $\tau= \pm 1$ depending on whether a diquark line or a single quark line starts the order) we can see that shifting mesons or baryonia from one cluster to another does not change the value of $\tau$ once the location of the dot is given. In short, only the cyclic order of the baryon and antibaryon variables is relevant for determining the value of $\tau$.

We now consider different terms or graphs in (2.1) in which the baryon and antibaryon clusters have been permuted. Suppose, e.g., we take as the first term in our sum the amplitude in Fig. 1. Then suppose we have another graph or term contributing to (2.1) as in Fig. 2, which has a common discontinuity with Fig. 1 but with the particle lines permuted. The dashed lines in Figs. 1 and 2 represent the variable in which the common discontinuity occurs. In Fig. 2, $P_{i}$ denotes some permutation of the lines $1,2,3$ and $P_{k}$ some permutation of the lines $\alpha, \beta, \gamma, \ldots$. (We shall consider here only permutations of the lines which are a product of two permutations for reasons that will become clear shortly.) We note that we are assuming that Fig. 2 and Fig. 1 correspond to the same process so we are assuming meson and baryonia states must be the same for each although some of these might be associated with different clusters in the two terms. The question is where should the dot go in Fig. 2 if it is to be added to Fig. 1 ?

To answer this question we shall invoke the basic requirement of the self-consistency of the zero-entropy amplitudes in satisfying a discontinuity equation of the form (2.1) of FJ. We begin by making several observations and remarks.

(i) Wherever the dot is placed in Fig. 2 we obviously will obtain an amplitude whose variables represent either an even or an odd permutation of those in Fig. 1 which have the order $(1,2,3, \ldots, \alpha, \beta, \gamma, \ldots)$. We shall see that adding amplitudes whose variables are even permutations of one another is the self-consistent choice.

(ii) All ways of inserting the dot in Fig. 2 in such a way that results in an even permutation of variables relative to Fig. 1 give the same amplitude. This is because moving the dot past an even number of baryon or antibaryon clusters does not change the amplitude. Moving the dot past any one cluster changes the $\tau$ factor by a minus sign so after an even number of steps one returns to the same amplitude. Similarly all ways of inserting the dot in Fig. 2 which results in an odd permutation of variables relative to Fig. 1 give the same amplitude.

We shall now show that adding zero-entropy ampli- 
tudes whose variables are even permutations of one another results in self-consistency with respect to the discontinuity equation (2.1). Afterwards, we shall indicate that other conceivable choices for the permutations in the sum are not self-consistent.

We have put the variables in Figs. 1 and 2 into two groups $(1,2, \ldots)$ and $(\alpha, \beta, \gamma, \ldots)$ corresponding to a common discontinuity for these two graphs indicated by the dotted lines. Although we have suppressed the meson and baryonium variables in these amplitudes we assume the same variables for these states are associated with the same two groups of variables for both Figs. 1 and 2 . We shall take the common discontinuity indicated above across the same intermediate state, which in the case of Fig. 1 we shall designate by the variables $(a, b, c, \ldots)$ and in the case of Fig. 2 by some permutation $P_{j}(a, b, c, \ldots)$ of these variables. These planar discontinuities are depicted in Figs. 3(a) and 3(b) for the individual terms, where we have arbitrarily placed a dot in Fig. 3 above the group of particle lines corresponding to the variables $P_{i}(1,2,3, \ldots)$. This dot will, in general, have to be moved when combining the discontinuity of Fig. 3(b) with that of Fig. 3(a), assuming the location of the dot in Fig. 3(a) to be fixed. Assuming that the correct prescription is to add amplitudes whose variables are even permutations of one another we would have for the two amplitudes of Figs. 1 and 2 the sum

$$
\begin{aligned}
& A(1,2,3, \ldots, \alpha, \beta, \gamma, \ldots) \\
& \quad+(-1)^{\delta_{i k}+1} A\left(P_{i}(1,2,3, \ldots), P_{k}(\alpha, \beta, \gamma, \ldots),\right.
\end{aligned}
$$

where the indices $i$ and $k$ are two-valued denoting either even or odd permutations (recall that moving the dot past a cluster produces a minus sign). The phase factor in front of the second term in (2.2) in effect designates where the dot should be placed in the graph of Fig. 2 when adding it to that of Fig. 1. This same factor $(-1)^{\delta_{i k}+1}$, of course, must also be inserted in the expression for the discontinuity of (2.2) which results from adding the discontinuities of Figs. 3(a) and 3(b). However, the discontinuity formula can also be written as a bilinear product of sums of other zero-entropy amplitudes for processes involving the intermediate states. The term in this bilinear product corresponding to the process (intermediate state $) \rightarrow$ (final state), where the final state is defined as
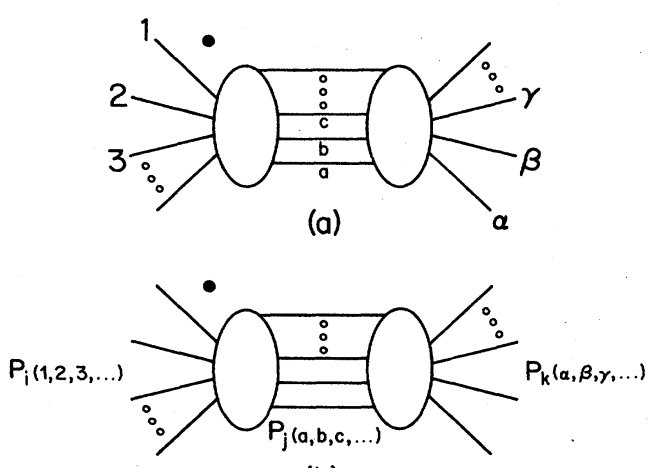

(b)

FIG. 3. Discontinuity graphs for Figs. 1 and 2. the one involving the $(1,2, \ldots)$ clusters is just the sum:

$$
\begin{aligned}
A_{+} & (1,2,3, \ldots, a, b, c, \ldots) \\
& +(-1)^{\delta_{i j}+1} A_{+}\left(P_{i}(1,2,3, \ldots) P_{j}(a, b, c, \ldots)\right)
\end{aligned}
$$

using the same even permutation rule as was assumed for the original amplitude and the plus sign refers to above the cut as in FJ. The term in the product corresponding to the process (initial state) $\rightarrow$ (intermediate state) is the sum:

$$
\begin{aligned}
& A_{-}(\ldots, c, b, a, \alpha, \beta, \gamma, \ldots) \\
& \quad+(-1)^{\delta_{j k}+1} A_{-}\left(P_{j}^{R}(\ldots, c, b, a) P_{k}(\alpha, \beta, \gamma, \ldots)\right),
\end{aligned}
$$

where $P_{j}^{R}(\ldots, c, b, a)$ is just the reverse of $P_{j}(a, b, c, \ldots)$, and the minus sign refers to below the cut. Clearly $P_{j}^{R}$ has the same evenness or oddness as a permutation as $P_{j}$ has. The self-consistency of taking even permutations ${ }^{9}$ in the zero-entropy sum is then simply proved by the observation:

$$
(-1)^{\delta_{i j}+1}(-1)^{\delta_{j k}+1}=(-1)^{\delta_{i k}+1} .
$$

It can be readily shown that the rule of adding amplitudes whose variables are even permutations of one another is also self-consistent in the special case where the $P_{k}$ permutation is the identity. In this situation there may be four terms in the expression for the discontinuity.

One cannot achieve self-consistency using a sum of two amplitudes whose variables are odd permutations of one another since the additive plus one in exponential of each factor in (2.5) would be missing and there would be no equality.

Having achieved self-consistency adding amplitudes in (2.1) with dots placed so that only even permutations of variables occur, we must now ask whether self-consistency can be achieved with any other rule for dot placement. We have seen that an odd permutation rule is not selfconsistent. The amplitudes we are adding only differ from one another by permutations of the variables so the rule-which must be general enough to work for any process-can only specify which relative permutations to use in generating the sum. We emphasize that the value of a given amplitude is determined once we specify whether it has an even or odd permutation of variables with respect to some reference amplitude. So the only question remaining is whether any self-consistent general rule can be given for summing zero-entropy amplitudes which can give a mixture of even and odd permutations of the variables.

The only rule of this type that can be given is one where, for example, the dot is placed in each zero amplitude at the same place with respect to a given cluster. Thus we might have a rule in which the dot is always placed adjacent clockwise to cluster 1. Rules of this type will produce in general a sum of zero-entropy amplitudes, some of which have an even permutation of variables with respect to some reference amplitude and others an odd permutation. Simple examples can be given, however, to show that such rules are not self-consistent with respect to the discontinuity relation. Thus in general the only self- 


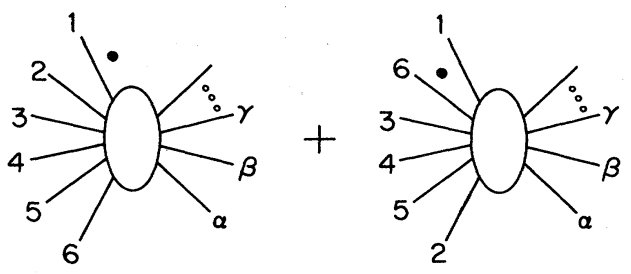

FIG. 4. Sum of graphs indicating Fermi statistics for baryons and antibaryons.

consistent rule is the even-permutation rule for the variables.

Finally we verify that our results in this section imply the normal Fermi statistics for baryons and antibaryons. We first note that the clusters alternate in their baryon number property: if cluster 1 contains an outgoing baryon or incoming antibaryon, then cluster 2 contains an incoming baryon or outgoing antibaryon, etc. Suppose now that two different clusters contain baryons (or antibaryons) of the same type with both either incoming or outgoing. For example, suppose clusters 2 and 6 have this property. Then, (2.1) will consist of sums of pairs of terms with 2 and 6 interchanged. One such pair is depicted in Fig. 4, where each graph is assumed to have the same spin-patch structure. The crucial point is that the dot must be relocated in the second term of Fig. 4 in order to have an even permutation of variables relative to the first term. Clearly if variables 2 and 6 are interchanged, we have introduced an odd permutation of the variables relative to the first term if the dot remains above cluster 1 . Thus to reinstate an even permutation of variables the dot must be advanced past an odd number of clusters as shown in Fig. 4. As discussed earlier the $\tau$ factor changes sign every time the dot is moved past a cluster so the two terms in Fig. 4 have opposite $\tau$ factors and the sum is antisymmetric under the interchange of momentum and spin variables for two identical baryons or antibaryons.

Thus we have proved that self-consistency requires the correct connection between spin and statistics for the sum of zero-entropy amplitudes corresponding to hadronic reactions. Since the correct connection between spin and statistics exists at the zero-entropy level, it will persist to all levels of the topological expansion-each level of topological complexity (such as cylinder terms, torus terms, etc.) separately exhibiting the correct connection between spin and statistics.

\section{PARITY INVARIANCE}

We shall now show that the sum of zero-entropy terms (2.1) which is the first approximation to hadronic amplitudes is invariant under parity transformations. The condition of parity invariance expressed in terms of scattering amplitudes takes the following form ${ }^{3}$ for the zero-entropy sum of (2.1):

$$
A(A \cdots K)=A(\widetilde{A} \cdots \widetilde{K}) e^{i \theta_{P}}
$$

where

$$
A(A \cdots K)=\sum_{\left\{z_{A} \cdots z_{K}\right\}} \sum_{i} A^{z_{A} \cdots z_{K}}\left(P_{i}(A \cdots K)\right),
$$

and $\widetilde{A}, \widetilde{B}, \ldots$ refers to states in which the threemomentum has its sign reversed $\mathbf{p}_{A} \rightarrow-\mathbf{p}_{A}$, etc., but the spin state is unchanged. Thus parity invariance requires the amplitudes for two processes in which all particles and spin states are the same but for which all threemomenta are reversed to be equal up to an overall phase. We shall prove that this is true for the zero-entropy sum (3.1b).

The parity properties of (3.1b) are made evident by fixing the permutation $i$ and summing only over the spinpatch structure $\left\{z_{A} \cdots z_{K}\right\}$. Specifically we shall show that the sum (3.1b) can be grouped into pairs of terms each satisfying (3.1a). Further the phase $e^{i \theta_{P}}$ for each pair is the same so (3.1a) holds for the complete sum of zero-entropy terms. The pairs we consider are those in which spin-patch structures are completely reversed. In the notation of FJ one such pair can be written

$$
\begin{aligned}
B^{z_{A} \cdots z_{K}}(A \cdots K)= & A^{z_{A} \cdots z_{K}}((A \cdots K)) \\
& +A^{i_{A} \cdots \dot{z}_{K}}((A \cdots K)) .
\end{aligned}
$$

Now we determine this same sum for the paritytransformed process, namely,

$$
\begin{aligned}
B^{z_{A} \cdots z_{K}}(\widetilde{A} \cdots \widetilde{K})=A^{z_{A} \cdots z_{K}}((\widetilde{A} \cdots \tilde{K})) \\
+A^{i_{A} \cdots i_{K}}((\widetilde{A} \cdots \widetilde{K})) .
\end{aligned}
$$

According to Eq. (2.2) of FJ each amplitude on the right side of (3.2) and (3.3) can be written as the product of three terms, e.g.,

$$
\begin{aligned}
A^{z_{1} \cdots z_{K}}(A \cdots K) & \\
& =\Gamma S^{z_{A} \cdots z_{K}}(A \cdots K) f\left(P_{A} \cdots P_{K}\right),
\end{aligned}
$$

where $\Gamma$ is a phase given by (9.9) of $\mathrm{FJ}$, the second term is the spin structure factor, and $f$ is an invariant scalar function so that

$$
f\left(P_{A} \cdots P_{K}\right)=f\left(\widetilde{P}_{A} \cdots \widetilde{P}_{K}\right) .
$$

The spin structure factors given in (2.3) of FJ as a sequence of Lorentz-invariant products of two-component spinors can readily be shown to satisfy the identity:

$$
S^{z_{A} \cdots z_{K}}(A \cdots K)=S^{\dot{z}_{A} \cdots \dot{z}_{K}}(\widetilde{A} \cdots \widetilde{K}) .
$$

Examination of the phase $\Gamma$ in (3.4) as given in full generality by (9.9) of FJ shows that $\Gamma$ consists of a set of factors which are common to every term in the zero-entropy sum plus the factors

$$
(-1)^{N_{L}^{+(P)}} \epsilon^{n(34, P)},
$$

which may introduce relative signs between the terms in the zero-entropy sum. In (3.7), $N_{L}^{+}(P)$ is the total number of "like" paraquark lines whose ends are either both "in" states or both "out" states; $n(34, P)$ is the number of paraquark lines within diquark lines involved in a baryon (or 
antibaryon) to baryonium transition. Recalling that $\epsilon= \pm 1$ and utilizing (3.5) and (3.6) we can conclude that

$B^{z_{A} \cdots z_{K}}(A \cdots K)=(-1)^{N_{L_{Q}} B^{z_{A}} \cdots z_{K}}(\widetilde{A} \cdots \widetilde{K})$,

$e^{i \theta_{P}}=(-1)^{N_{\mathrm{LQ}}}$,

where $N_{\mathrm{LQ}}$ is the number of like quark lines in the graph whether these quark lines are ortho or para. While proved for a pair of terms with a given permutation of the variables, (3.8) clearly holds for any permutation of the variables. What may not be entirely clear is that $(-1)^{N_{\mathrm{LQ}}}$ has the same value for any permutation of the variables. This must be the case if (3.1) is to hold for the total zeroentropy amplitude.

To show that the factor $(-1)^{N_{\text {LQ }}}$ depends only on the overall process and not on the permutation of variables of a given term in the zero-entropy sum, we note the identity

$$
(-1)^{N_{\mathrm{LQ}}}=(-1)^{N_{Q}}=(-1)^{N_{\bar{Q}}},
$$

where $N_{Q}$ is the total number of quarks involved in the process whether incoming or outgoing and similarly $N_{\bar{Q}}$ is the total number of antiquarks. This result already shows that this phase factor is dependent only on the process and is the same for every pair of terms of the form (3.2).

Further insight occurs by noting the further identity

$$
(-1)^{N} Q=(-1)^{N(M)+N(B \bar{B})},
$$

where

$$
\begin{aligned}
N(M)= & \text { number of mesons in the amplitude, } \\
N(B \bar{B})= & \text { number of baryon-antibaryon pairs } \\
& \text { either incoming or outgoing in the } \\
& \text { amplitude. }
\end{aligned}
$$

The result of (3.11) is, of course, related to the fact that a negative intrinsic parity can be assigned to low-lying mesons and to baryon-antibaryon pairs.

The entire zero-entropy sum (3.1b) can be written as a sum over pairs of terms like (3.2), each pair satisfying a relation like (3.9) with the same phase $(-1)^{N_{\mathrm{LQ}}}$. Thus the entire zero-entropy sum satisfies (3.1) with

$$
e^{i \theta_{P}}=(-1)^{N_{\mathrm{LQ}}}=(-1)^{N(M)+N(B \bar{B})} .
$$

This completes the proof of parity invariance for hadronic processes at the zero-entropy level. As connected sums of the zero-entropy amplitudes are made to calculate higher-order approximation to the hadronic amplitudes, parity invariance will continue to hold at each level of the topological expansion involving purely hadronic processes.

\section{TIME-REVERSAL INVARIANCE}

In this section we shall show that the sum of zeroentropy terms is invariant under a time-reversal transformation. The discussion here parallels that given for parity invariance in the preceding section and, in particular, is again based on proving the invariance separately for pairs of terms of the form (3.2). The time-reversal transformation for a process involves

(a) reversing the three-momentum of each particle $\mathbf{p} \rightarrow-\mathbf{p}$;

(b) reversing all spin states;

(c) making all incoming particles outgoing and vice versa. We shall designate by the notation

$$
[A(A \cdots K)]_{\mathrm{TR}}
$$

any zero-entropy or sum of zero-entropy amplitudes in which the time-reversal transformation specified by (a)-(c) has been carried out on the process specified by the amplitude or sum of amplitudes within the square brackets.

If $A(A \cdots K)$ above designates the complete zeroentropy sum time-reversal invariance would imply

$$
[A(A \cdots K)]_{\mathrm{TR}}=e^{i \theta_{\mathrm{TR}}} A(A \cdots K) .
$$

To see if (4.1) holds for the zero-entropy sum we first examine the behavior of the spin structure factors $S^{z}$ under time reversal. These factors are made up of Lorentz-invariant scalar products of two-component spinors. An example of such a spinor is $\eta^{\dot{\alpha}}(v, \phi)$, whose Lorentz transformation properties are of the "upperdotted" variety. Here $v$ is the associated particle fourvelocity and $\phi$ is the two-component rest-frame spin state. As explained in Ref. 10 the spinor with a reversed spin direction is just

$$
\eta^{\dot{\alpha}}\left(v, C \phi^{*}\right)
$$

where

$$
C=-i \sigma_{2},
$$

and $\sigma_{2}$ is the standard Pauli matrix. Reversing the direction of the three-momentum converts the spinor in (4.2a) to

$$
\eta^{\dot{\alpha}}\left(\widetilde{v}, C \phi^{*}\right)
$$

Changing in states to out states converts (4.2b) into a spinor with a lower dotted index and also complex conjugates the rest-frame spin state giving

$$
\eta_{\dot{\alpha}}(\widetilde{v}, C \phi)=C \eta_{\alpha}(v, \phi) \text {. }
$$

The equality (4.3) also holds when the spinors have upper indices.

The above results on the change of the spinors when the time-reversed process is considered imply for a given spin structure factor:

$$
S^{z_{A} \cdots z_{K}}(A \cdots K)_{\mathrm{TR}}=S^{\dot{z}_{A} \cdots \dot{z}_{K}}(A \cdots K),
$$

where ( ) TR means that each of the variables in the original spin structure factors is time reversed as described in (a)-(c) above.

We now show that pairs of terms of the form (3.2) in the zero-entropy sum separately satisfy time-reversal invariance. Unlike the parity transformation, however, the time-reversal transformation takes us to a new process where in and out states have been reversed. Using (4.4) and (9.9) from FJ we find that the time-reversed ampli- 
tude for a given term in the zero-entropy sum is related to a zero-entropy amplitude for the original process by the relation
$A^{z_{1} \cdots z_{K}}(A \cdots K)_{\mathrm{TR}}=e^{i \theta_{\mathrm{TR}}} A^{\dot{z}_{1} \cdots \dot{z}_{K}}(A \cdots K)$,

where, in the notation of FJ Sec. IX,

$$
\begin{aligned}
e^{i \theta_{\mathrm{TR}}}= & (-1)^{N_{\mathrm{LQ}}}(-1)^{N(M)+N(\bar{B})} \exp \left[2 i\left(\gamma_{1}-\gamma_{2}\right)\left(N_{\text {out }}(M)-N_{\text {in }}(M)\right)\right] \\
& \times \exp \left[2 i\left(\gamma_{2}+\Gamma_{1}^{+}-\gamma_{4}-\Gamma_{3}^{+}\right)\left(N_{\text {out }}(B)-N_{\text {in }}(B)\right)\right] \exp \left[2 i\left(\Gamma_{2}^{+}-\Gamma_{4}^{+}\right)\left(N_{\text {out }}(B)-N_{\text {in }}(B)\right)\right] .
\end{aligned}
$$

By way of explaining the meaning of (4.5) and (4.6) we make the following remarks:

(i) In the time-reversed process in and out become interchanged so the variables have the opposite order to the original process, namely, $(K \cdots A)$. The $\tau$ factor with this order of variables for the time-reversed process is identical to that for the original process where the order of variables is $(A \cdots K)$.

(ii) All the variables such as $N_{\text {out }}(B)$ in (4.6) refer to the original process. $M(M)$ and $N(\bar{B})$ denote, respectively, the total number of mesons and antibaryons whether in or out.

(iii) Since it was shown in the previous section that the factor $(-1)^{N_{L Q}}$ depends only on the overall process and not on the particular zero-entropy expansion, it follows that the total phase $e^{i \theta_{\mathrm{TR}}}$ depends only on the process and not on which zero-entropy term we are time reversing.

From this discussion and (4.5) we can see that for the pair of terms (3.2) we have

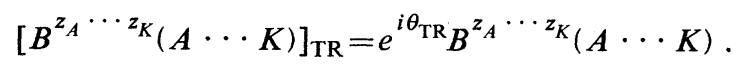

Since the same phase factor occurs for each pair of terms in the zero-entropy sum, (4.1) must hold and the entire zero-entropy sum is thus time-reversal invariant. This completes the proof of time-reversal invariance for hadronic processes at the zero-entropy level. As in the case of parity invariance, time-reversal invariance will continue to hold separately at each level of the topological expansion for purely hadronic processes.

\section{CHARGE-CONJUGATION INVARIANCE}

Here we shall prove that the zero-entropy amplitudes are invariant under charge-conjugation transformations. We shall show that each individual term in the zeroentropy sum is charged-conjugation invariant. This is unlike the cases of parity and time-reversal invariance where the invariance was evident only when we considered pairs of terms. In particular, the part of the phase of the amplitudes which depends on the spin-patch structure plays no role in establishing charge-conjugation invariance as it did in the case of parity and time reversal.

The charge-conjugation transformation involves

(a) changing quarks to antiquarks and vice versa;

(b) leaving spin states unchanged;

(c) leaving momenta unchanged;

(d) leaving in and out unchanged. The above description of the charge-conjugation transformation requires some elaboration. By (b) is meant that a quark present in the charge-conjugated process will be in the same spin state as the corresponding antiquark in the original process, etc. In carrying out (a) the quark lines in graphs denoting the zero-entropy terms must have their directions reversed. But to maintain our convention (see FJ) that single quark lines run clockwise and diquark lines run counterclockwise we must "flip" the graph for the charge-conjugated process leading to a reverse cyclic order for the variables. This is illustrated for a sample graph in Fig. 5, where $\mathscr{C}$ denotes charge conjugation for the particular zero-entropy graph.

The key observation in proving charge-conjugation invariance is that a two-component spinor such as $\eta^{\dot{\alpha}}(v \phi)$ associated with the tail of one of the quark lines in the graph becomes replaced in the charge-conjugated process by

$$
\eta^{\dot{\alpha}}(v, \phi) \rightarrow \eta_{\dot{\alpha}}(v, C \phi)
$$

The lowering of the index in (5.1) just means a quark is replaced by an antiquark or vice versa. As explained in Ref. 10, the transformation of the rest-frame spin state $\phi \rightarrow C \phi$ is necessary in order that the antiquark (quark) be in the same spin state as the original quark (antiquark). The need for this transformation of the rest-frame spin state quark and antiquark states transform according to different representations of the rotation group (these representations, in fact, are just complex conjugations of each other). The transformation $\phi \rightarrow C \phi$ in (5.1) is appropriate for all spinors in the spin structure factor for the chargeconjugated process whether the spinor has upper or lower, dotted or undotted indices.

We shall now show, taking one term of the zeroentropy sum that

$$
A^{z_{A} \cdots z_{K}}(A \cdots K)=e^{i \theta_{c}} A^{z_{K} \cdots z_{K}}(\bar{K} \cdots \bar{A}),
$$

where an example of two amplitudes such as those on the left and right side of (5.2) is given graphically in Fig. 5. It is understood in (5.2) that the spin states are the same on both sides of the equation as explained above. We shall

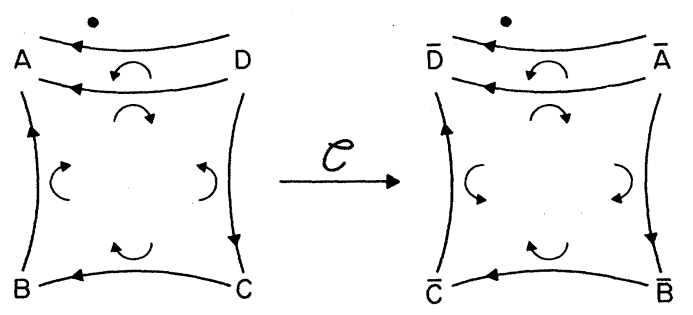

FIG. 5. Effect of charge conjugation on amplitude. 
find that (5.2) holds for each term in the sum (2.1) with the same phase $e^{i \theta_{c}}$ so that a relation of the form (5.2) holds for the entire zero-entropy sum. This means the theory is charge-conjugation invariant.

We have already discussed how the individual spinors change under charge conjugation. The spin structure factor is made up of Lorentz-invariant spinor products of the form

$$
\eta_{\dot{\alpha}}\left(v^{\prime}, \phi^{\prime}\right) \eta^{\dot{\alpha}}(v, \phi)
$$

In the charge-conjugated process this scalar product becomes

$$
\eta^{\dot{\alpha}}\left(v^{\prime}, C \phi^{\prime}\right) \eta_{\dot{\alpha}}(v C \phi)=\eta_{\dot{\alpha}}\left(v^{\prime}, \phi^{\prime}\right) \eta^{\dot{\alpha}}(v, \phi)
$$

which shows that the spin structure factor is invariant under charge conjugation. The invariant scalar function $f$ in (3.4) does not change under this transformation (it does not depend on flavor-see Ref. 10). To determine the phase in (5.2), we examine the amplitude phases given by (9.9) of FJ. The result is simply

$$
e^{i \theta_{c}}=(-1)^{N_{\mathrm{in}}(B)+N_{\mathrm{in}}(\bar{B})},
$$

where the sum of the two integers in the right-hand exponent can be evaluated either for the original or the charge-conjugated process since they have the same value. It is clear the phase (5.5) will be the same for each term in the zero-entropy sum (2.1). It is important to note that when the left side of (5.2) is summed over all the appropriate even permutations of the variables $(A \cdots K)$ as discussed in Sec. II, the right side will automatically come out as an appropriate sum of the even permutations of $(\bar{K} \cdots \bar{A})$ all multiplied by the common phase $e^{i \theta_{c}}$.

This completes the proof of charge-conjugation invariance in hadronic processes. As in the other cases this invariance will continue to hold at each level of the topological expansion for hadronic processes.

\section{DISCUSSION}

It has long been the goal of $S$-matrix theory to start from general basic assumptions-including Lorentz invariance, crossing analyticity-and to deduce from selfconsistency the properties and parameters of elementary particle interactions that must be assumed in other more conventional approaches. We have shown here that topological particle theory-the modern version of $S$-matrix theory-is capable of explaining the separate discrete invariances of parity, time reversal, and charge conjugation in strong interactions as well as the connection between spin and statistics.

Earlier work in $S$-matrix theory ${ }^{3}$ showed that the postulates of the theory implied the correct connection between spin and statistics and the invariance under the product of the above three discrete symmetries. The new topological theory, however, clearly separates the origins of the strong-interaction terms associated with hadronic processes and the terms associated with electroweak processes. $^{4-6}$ The former come from the zero-entropy terms in the topological expansion ${ }^{7,8}$ involving coupling strengths known to be of the correct order of magnitude for strong interactions. ${ }^{11}$ Terms responsible for electroweak ${ }^{4-6}$ processes do not occur at the zero-entropy level.

Owing to this unambiguous identification of zeroentropy with strong interactions, it has been possible here to prove the existence of the three separate discrete invariances for such processes. These separate invariances, of course, fail in the terms of the topological expansion associated with electroweak processes. Thus topological particle theory gives a basis for answering the age-old question of why the degree of symmetry in interactions is associated with the strength of the interaction, at least in the case of the discrete symmetries.

\section{ACKNOWLEDGMENT}

This work was supported in part by National Science Foundation Grant No. PHY-8308053.
${ }^{1}$ P. Finkler and C. E. Jones, preceding paper, Phys. Rev. D 31, 1393 (1985).

${ }^{2}$ H. P. Stapp, Phys. Rev. 125, 2139 (1962).

${ }^{3}$ H. P. Stapp, Phys. Rev. 128, 1963 (1962).

${ }^{4}$ G. F. Chew and J. Finkelstein, Phys. Rev. Lett. 50, 795 (1983).

${ }^{5}$ G. F. Chew and V. Poénaru, Z. Phys. C 14, 233 (1982).

${ }^{6}$ G. F. Chew, J. Finkelstein, R. M. McMurray, Jr., and V. Poénaru, Phys. Lett. 100B, 53 (1981); Phys. Rev. D 24, 2287
(1981).

${ }^{7}$ H. P. Stapp, Phys. Rev. D 27, 2445 (1983); 27, 2478 (1983).

${ }^{8}$ G. F. Chew and V. Poénaru, Z. Phys. C 11, 59 (1981).

${ }^{9}$ This prescription appears to be identical to the one assumed by Stapp (Ref. 7).

${ }^{10}$ C. E. Jones and J. Uschersohn, Phys. Rev. D 27, 366 (1983).

${ }^{11}$ G. F. Chew, J. Finkelstein, and M. Levinson, Phys. Rev. Lett. 47, 767 (1981). 